\title{
TRANSFINITE RATIONALS
}

\author{
JOHN M. H. OLMSTED
}

The standard method of extending a system with an operation to a system with the operation and its inverse depends heavily on the law of cancellation: $a b=a c$ implies $b=c$ (possibly with some limitation on $a$ ). The most familiar example is undoubtedly the extension of the integers to the rational numbers. If the law of cancellation fails, not only does the method fail, but the extension cannot be a group. However, one may ask whether some method of extension is available in certain cases where the cancellation law fails but is replaced by something else, for example an order relation. It is the purpose of this paper to present an extension of the system of cardinal numbers (the positive cardinals, excluding zero). This will be an extension of both the cardinal numbers and the positive rational numbers with respect to the operations of addition and multiplication and the relation of ordering. Furthermore, this extension is the smallest extension subject to certain conditions. We shall assume the axiom of choice in the form of the simple ordering of the cardinals.

The means for obtaining the extension is suggested by a treatment of ratios by Eudoxus. (See for instance E. T. Bell, Development of mathematics, New York, 1940, p. 61.)

We define first an equivalence relation between ordered pairs of cardinal numbers: $(a, b) \rho(c, d)$ if and only if (1) $a>b$ and $c>d$, or (2) $a=b$ and $c=d$, or (3) $a<b$ and $c<d$.

In terms of the relation $\rho$ we define the fundamental equivalence relation between ordered pairs of cardinal numbers:

Definition. $(a, b) \sim(c, d)$ if and only if for every pair of cardinal numbers, $m$ and $n,(m a, n b) \rho(m c, n d)$.

The relation $\sim$ is readily shown to be an equivalence relation. Before discussing the equivalence classes defined by this relation we shall state a theorem, assuming for the first two parts that $\left(a_{1}, b_{1}\right) \sim\left(a_{2}, b_{2}\right)$.

THEOREM. I. If $a_{1}$ and $b_{1}$ are finite so are $a_{2}$ and $b_{2}$. II. If $a_{1}<b_{1}$ and $b_{1}$ is infinite, then $b_{1}=b_{2}$. If $a_{1}>b_{1}$ and $a_{1}$ is infinite, then $a_{1}=a_{2}$. III. If $a_{1}<b, a_{2}<b$, and $b$ is infinite, then $\left(a_{1}, b\right) \sim\left(a_{2}, b\right)$. If $a>b_{1}$, $a>b_{2}$, and $a$ is infinite, then $\left(a, b_{1}\right) \sim\left(a, b_{2}\right)$. IV. If $(a, a) \sim(b, b)$ and $a$ and $b$ are infinite, then $a=b$.

Presented to the Society, September 17, 1945; received by the editors February 12, 1945, and, in revised form, April 9, 1945. 
As a sample proof we shall prove the first half of part II. Choose $m=b_{1}, n=1$. Since $b_{1} a_{1}=1 \cdot b_{1}$, therefore $b_{1} a_{2}=1 \cdot b_{2}$. But $a_{2}<b_{2}$, and therefore $b_{1}=b_{2}$.

Each equivalence class $\{(a, b)\}$ defined by the relation $\sim$ will be called a rational, and the entire set will be denoted by $R$. If $a$ and $b$, for any equivalence class representative, are finite, the class will be called a finite rational; otherwise, a transfinite rational. We shall now define ordering and multiplication of rationals. In each case it is necessary to show that the definition is independent of the representatives chosen. The proofs for the two cases are similar, and only that for multiplication will be given.

DEFINITION OF ORDERING. $\{(a, b)\} \leqq\{(c, d)\}$ if and only if for every pair of cardinal numbers, $m$ and $n$,

$m a>n b \quad$ implies $m c>n d, \quad m a=n b$ implies $m c \geqq n d$.

Remarks. 1. The definition of $\leqq$ is independent of the equivalence class representatives. 2. If $\{(a, b)\}=\{(c, d)\}$, then $\{(a, b)\} \leqq\{(c, d)\}$. 3. If $\{(a, b)\} \leqq\{(c, d)\}$ and $\{(a, b)\} \geqq(c, d)\}$, then $\{(a, b)\}$ $=\{(c, d)\}$. 4. $\{(a, b)\} \leqq\{(c, d)\}$ if and only if $\{(b, a)\} \geqq\{(d, c)\}$. 5 . The relation $<$ is defined in terms of $\leqq$ and $=$. 6 . The rationals form a partially ordered system.

Definition of MULtiplication. $\{(a, b)\} \cdot\{(c, d)\}=\{(a c, b d)\}$.

Remarks. 1. $\left(a_{1}, b_{1}\right) \sim\left(a_{2}, b_{2}\right)$ implies $\left(a_{1} c, b_{1} d\right) \sim\left(a_{2} c, b_{2} d\right)$. [In order to show that $\left(m a_{1}, n b_{1}\right) \rho\left(m a_{2}, n b_{2}\right)$ implies $\left(q a_{1} c, r b_{1} d\right) \rho\left(q a_{2} c, r b_{2} d\right)$, let $m=q c$ and $n=r d$.] 2. $\left(a_{1}, b_{1}\right) \sim\left(a_{2}, b_{2}\right)$ and $\left(c_{1}, d_{1}\right) \sim\left(c_{2}, d_{2}\right)$ imply $\left(a_{1} c_{1}, b_{1} d_{1}\right) \sim\left(a_{2} c_{2}, b_{2} d_{2}\right)$. [ $\left(a_{1} c_{1}, b_{1} d_{1}\right) \sim\left(a_{2} c_{1}, b_{2} d_{1}\right) \sim\left(a_{2} c_{2}, b_{2} d_{2}\right)$.] 3. Multiplication is commutative and associative. $4 .\{(a, b)\}=\{(a c, b c)\}$ only if $c$ is not too large.

In order to imbed the cardinals in $R$ we define the correspondence $a \rightleftarrows\{(a, 1)\}$. It is readily shown that this correspondence is one-one, preserves ordering, and is isomorphic with respect to multiplication. Furthermore, the definitions of ordering and multiplication in the case of the finite rationals are equivalent to those normally given for rational numbers. The finite rational $\{(1,1)\}$ is a multiplicative unit for the entire system $R$.

Adopting the notation $a / b$ for $\{(a, b)\}$, letting $f$ represent any finite rational, and using $x$ and $y$ to represent any two transfinite cardinals where $x<y$, we can indicate the order structure of $R$ by means of an abbreviated Hasse diagram : 


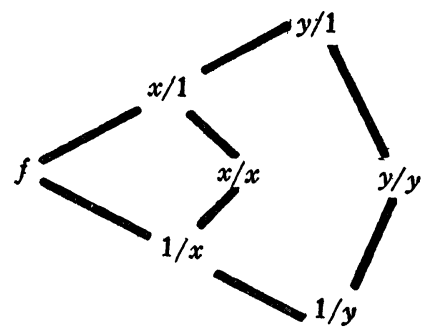

The complete diagram would contain three sequences of transfinite rationals radiating from $f$. From the above diagram it can be seen that $R$ is a non-modular lattice. It might be noted that the product of any two rationals, not both finite, is represented on the diagram by the one which is farther to the right, unless the two are distinct and situated on a common vertical, in which case the product is the first element to the right of both. (One could obtain a new ordering by giving the complete Hasse diagram a quarter turn, the result being a distributive lattice where multiplication is identical with the lattice operation $U$. In this case it is to be understood that only one finite rational is included.)

Definition OF DIVISION. $a / b \div c / d=a d / b c$.

Since $a / 1 \div b / 1=a / b$, the notation $A / B$ will be used for quotients of elements of $R$ as well as for equivalence classes of pairs, without danger of confusion. Although many familiar laws involving division persist, let us observe that multiplication and division are not inverse operations in the usual sense. For example, a rational divided by itself is the unit element only if the rational is finite.

The definition of addition which first comes to mind is $a / b+c / d$ $=(a d+b c) / b d$, particularly since it can be shown that this is independent of the representatives chosen. However, this possibility will be rejected for two reasons. In the first place, the expected distributive law fails, and secondly the corresponding formula for adding quotients of elements of $R$ fails. A more satisfactory method is to impose certain requirements and prove that the resulting operation exists and is unique. To furnish a more concise arrangement of material, a third method has been chosen which is equivalent to the second. The definition will be given in a form which is apparently arbitrary, but it will be justified by properties given subsequently.

Definition of addition. $A+B=A \cup B$ unless $A$ and $B$ are both finite rationals, in which case the sum corresponds to the sum of the corresponding rational numbers. 
We now state and prove the principal theorem which enumerates properties of $R$ and establishes its minimal character.

THEOREM. The following conditions are satisfied by the system of rationals $R$. Furthermore, $R$ can be imbedded isomorphically with respect to the operations of multiplication, addition, division, $\cup$, and $\cap$ in any lattice $L$ with operations of multiplication, addition, and division defined, subject to the following conditions: I. $L$ is an extension of the positive rational numbers and the cardinals with respect to addition, multiplication, division, and countable $\cup$ and $\cap$, wherever these are defined among the positive rational numbers and the cardinals. II. Addition and multiplication are commutative and associative. The following distributive laws hold: multiplication over addition, over $\cup$, and over $\cap$; addition over $\cup$. III. There exists a unit $I$ such that $A I=A / I=A$. IV. $(A / B)(C / D)=(A / B) /(D / C)=A C / B D$. V. $A<B$ implies $I / A>I / B$. VI. $I<A<B$ implies $A / B<I$. VII. $A \leqq A+B$.

Proof that $R$ actually has the properties claimed will be omitted, since there is no difficulty present. Proof of the distributive laws consists of verification of all cases, but this is simplified by the partial equivalence between addition and $\cup$, and the duality between $U$ and $\cap$ where multiplication is involved.

Assume now that $L$ is a lattice with the given properties. The distributive laws for addition and multiplication over $\cup$ will be used in the forms: $\mathrm{A}<B$ implies $A+C \leqq B+C$ and $A C \leqq B C$. For the sake of compactness the following notational conventions will be made: the letter $F$ will denote an element of $L$ corresponding to a positive rational number, $P$ an element corresponding to a positive integer, and $T, X$, and $Y$ elements corresponding to transfinite cardinals with the added assumption that $X<Y$. Proof that $L$ is an extension of $R$ is divided into four parts.

I. Ordering. 1. By property I, $P<T .2 . F<T$, since $F$ is less than some $P$. 3. By property $\mathrm{V}, I / Y<I / X<F<X<Y$. 4. By property $\mathrm{V}$ and the fact that $T / T$ is not a multiplicative unit, there can be no order relation between $I$ and $T / T$. 5. By property VI, $I<Y / X$. Since multiplication is distributive over $\cup, I<Y / X \leqq Y / I=Y$. Assume $I<Y / X<Y$. Then by property VI, $(Y / X) / Y=Y / Y<I$. Since this is impossible, $Y / X=Y$. 6. Similarly, $T / F=F T=T$ and $F / T=I / T$. 7. By property $\mathrm{V}$, there is no order relation between $X / X$ and $Y / Y$ unless they are equal. Assume $Y / Y=X / X$. Then $(Y / Y)(Y / X)$ $=(X / X)(Y / X)$ or $Y / Y=Y / X>I$. This is impossible. 8. Similarly, there can be no order relation between $X$ and $Y / Y$, or between $I / X$ and $Y / Y$. 9. $I / T<T / T<T$. In establishing these order relations be- 
tween quotients of certain pairs of elements of $L$, we have incidentally proved the more fundamental fact that the obvious imbedding correspondence is one-one. For example, the distinct elements $x / 1,1 / y$, and $y / y$ of $R$ correspond to distinct elements $X, I / Y$, and $Y / Y$ of $L$.

II. Multiplication and division. Proof is trivial by property IV and by what has been proved in part I.

III. Addition. A sample collection of pairs of quotients of elements of $L$ will be chosen, and the sum of each pair determined. 1. $T \leqq F+T \leqq T+T$. By property $\mathrm{I}, T+T=T$, and therefore $F+T$ $=T$. 2. By a distributive law, $I / T+I / T=(I+I) / T=F / T=I / T$. More generally, any element of $L$ corresponding to a transfinite rational is idempotent with respect to addition as well as multiplication, $\cup$, and $\cap$. 3. It follows from the inequalities $T / T \leqq I / T+T / T$ $\leqq T / T+T / T=T / T$ that $I / T+T / T=T / T$. 4. Assume $I=I+T / T$. Multiplication by $I / T$ gives $I / T=I / T+T / T=T / T$. This is false, and therefore, since $I \leqq I+T / T, I<I+T / T$. 5. Since $I<T$ and $T / T<T, I+T / T \leqq T+T=T$. Assume $I<I+T / T<T$. Then by property VI, $(I / T)(I+T / T)<I$, or $I / T+T / T=T / T<I$. This is impossible, and therefore $I+T / T=T$. 6. $F+T / T=F(I+T / T)=F T$ $=T$. 7. $X / X+Y / Y=(X / X)(I+Y / Y)=(X / X)(Y)=Y$. 8. $F+I / T$ $\leqq F+F^{\prime}$, where $F$ and $F^{\prime}$ correspond to arbitrary finite rationals. From property $\mathrm{I}$, since $\cap$ [all finite rationals $>f]=f$, it follows that $\bigcap_{F^{\prime}}\left[F+F^{\prime}\right]$ exists and equals $F$. Therefore $F \leqq F+I / T \leqq F$, and $F+I / T=F$.

IV. $\cup$ and $\cap$. In $R$ countable $\cup$ and $\cap$ are trivial except where finite rationals are concerned, and the isomorphism proof in this case is a consequence of property I. Finite $U$ and $\cap$ cause no difficulty.

University of Minnesota 